\title{
ON THEOREMS OF TYCHONOFF, ALEXANDER, AND R. RADO
}

\author{
E. S. WOLK
}

It is well known [4], [7] that the Tychonoff theorem on products of compact spaces is equivalent to the axiom of choice (AC). It is therefore natural to consider Tychonoff's theorem for products of finite spaces as a restricted form of $\mathrm{AC}$, and to ask whether there are other restricted versions of $\mathrm{AC}$ to which it is equivalent. In this note we shall show that, assuming the axiom of choice for families of finite sets $(\mathrm{ACF})$, the following three theorems are all equivalent.

THEOREM TF. If $\left\{X_{i}: i \in I\right\}$ is a family of nonempty finite spaces, then $\Pi\left\{X_{i}: i \in I\right\}$ is compact in its product topology.

Theorem R (R. RAdo [5]). Let $\left\{X_{i}: i \in I\right\}$ be a family of nonempty finite sets, and suppose that for each finite subset $A$ of $I$ we are given a choice function $f_{A}$ for the family $\left\{X_{i}: i \in A\right\}$. Then there exists a choice function $f$ for $\left\{X_{i}: i \in I\right\}$ such that, whenever $A$ is a finite subset of $I$, there is a finite set $B$ with $A \subseteq B \subseteq I$ and $f(i)=f_{B}(i)$ for all $i \in A$.

Theorem A (Alexander Subbase Theorem). A topological space $X$ is compact if there exists a subbase $S$ for the closed sets of $X$ such that every subfamily of $\delta$ with f.i.p. has a nonvoid intersection.

The proposition ACF may be stated as follows.

Aхгом ACF. There exists a choice function for every family of nonempty finite sets.

The implication $\mathrm{TF} \rightarrow \mathrm{R}$ has already been proved by Gottschalk [2]. We shall demonstrate that $R \rightarrow A$. The implication $A \rightarrow T F$ is a standard argument, and for finite spaces involves only $A C F$ : the reader is referred to [3, p. 143] for details.

Our proof that $\mathrm{R} \rightarrow \mathrm{A}$ resembles the argument of Alexander [1] and Wallman [6, pp. 123-124], except that we use Theorem $R$ as a substitute for transfinite induction. Also, we do not use the theorem "A topological space $X$ is compact if there is a base $B$ for the closed sets of $X$ such that every subfamily of $B$ with f.i.p. has a nonvoid intersection"; since the usual proof of this theorem involves the unrestricted axiom of choice (although this use of $\mathrm{AC}$ can be avoided).

Received by the editors July 1, 1966. 
If $\mathcal{F}$ is any family of sets, for notational convenience we shall write $\cup \mathfrak{F}$ and $\cap \mathcal{F}$ for $U\{S: S \in \mathfrak{F}\}$ and $\cap\{S: S \in \mathcal{F}\}$, respectively.

To prove Theorem A, let $X$ be a topological space, and suppose that $S$ is a sub-base for the closed sets of $X$ such that every subfamily of $\delta$ with f.i.p. has a nonvoid intersection. Let $g$ be any family of closed subsets of $X$ with f.i.p. We must show that $\cap \mathcal{F} \neq \varnothing$. Define

$\Gamma=\{\mathfrak{F}: \mathcal{F}$ is a finite subfamily of $\delta$ and $\bigcup \mathfrak{F}$ contains a member of $\mathcal{G}\}$,

$B=\{B: B=\bigcup \mathfrak{F}$ for some $\mathfrak{F} \in \Gamma\}$.

We assert that $\cap B \subseteq \cap \mathcal{G}$. For suppose that $x \notin \cap \mathcal{G}$. Then there exists $A \in \mathcal{G}$ with $x \notin A$. Since $\mathcal{S}$ is a subbase, there is a finite subfamily $\mathfrak{F}$ of $\delta$ with $A \subseteq \bigcup F$ but $x \notin U F$. Hence $x \notin \cap ß$. Thus it will be sufficient to prove $\bigcap_{B \neq \varnothing}$.

We shall apply Theorem $\mathrm{R}$ to the family $\Gamma$. To this end, let $\Phi=\left\{\mathscr{F}_{1}, \mathfrak{F}_{2}, \cdots, \mathfrak{F}_{k}\right\}$ be any finite subset of $\Gamma$. For $i=1, \cdots, k$, let us write

$$
\begin{aligned}
\mathcal{F}_{i} & =\left\{S_{i 1}, S_{i 2}, \cdots, S_{i n_{i}}\right\}, \\
B_{i} & =\bigcup \mathcal{F}_{i}=S_{i 1} \cup S_{i 2} \cup \cdots \cup S_{i n_{i}} .
\end{aligned}
$$

Since the family $\&$ has f.i.p., we have $\cap\left\{B_{i}: i=1, \cdots, k\right\} \neq \varnothing$. Now let $\Lambda$ denote the set of all $k$-tuples $\lambda=\left(\lambda_{1}, \lambda_{2}, \cdots, \lambda_{k}\right)$, where $1 \leqq \lambda_{i}$ $\leqq n_{i}$ for all $i=1, \cdots, k$. Then

$$
\bigcap_{i=1}^{k} B_{i}=\bigcap_{i=1}^{k}\left[\bigcup_{j=1}^{n_{i}} S_{i j}\right]=\bigcup_{\lambda \in \Delta}\left[S_{1 \lambda_{1}} \cap S_{2 \lambda_{2}} \cap \cdots \cap S_{k \lambda_{k}}\right] .
$$

Now $\cap\left\{B_{i}: i=1, \cdots, k\right\} \neq \varnothing$ implies

$$
S_{1 \lambda_{1}} \cap S_{2 \lambda_{2}} \cap \cdots \cap S_{k \lambda_{k}} \neq \varnothing \quad \text { for some } \lambda \in \Lambda .
$$

Hence we may define a choice function $f_{\Phi}$ for the family $\Phi$ $=\left\{F_{1}, \cdots, \mathfrak{F}_{k}\right\}$ by

$$
f_{\Phi}\left(\mathcal{F}_{i}\right)=S_{i \lambda_{i} \in \mathcal{F}_{i}},
$$

where $\lambda=\left(\lambda_{1}, \cdots, \lambda_{k}\right)$ is chosen so that

$$
\cap\left\{S_{i \lambda_{i}}: i=1, \cdots, k\right\} \neq \varnothing .
$$

We may choose such a $\lambda \in \Lambda$ for each finite $\Phi \subseteq \Gamma$ by ACF.

By Theorem $\mathrm{R}$ there exists a choice function $f$ for the family $\Gamma$ such that, whenever $\Phi$ is a finite subset of $\Gamma$, there is a finite $\Psi$ with $\Phi \subseteq \Psi$ $\subseteq \Gamma$ and $f(\mathcal{F})=f_{\Psi}(\mathcal{F})$ for all $\mathfrak{F} \in \Phi$. We assert that the family $\{f(\mathcal{F})$ : $\mathscr{F} \in \Gamma\}$ has f.i.p. For given any finite $\Phi \subseteq \Gamma$, there is a finite $\Psi$ with $\Phi \subseteq \Psi \subseteq \Gamma$ and $\cap\{f(\mathcal{F}): \mathscr{F} \in \Phi\}=\bigcap\left\{f_{\Psi}(\mathfrak{F}): \mathfrak{F} \in \Phi\right\} \neq \varnothing$. Since $f(\mathfrak{F}) \in \mathcal{S}$ for all $\mathfrak{F} \in \Gamma$, the hypothesis of Theorem $A$ applies and we conclude 
that $\cap\{f(\mathfrak{F}): \mathfrak{F} \in \Gamma\} \neq \varnothing$. But then $\cap \Re \neq \varnothing$; for $y \in f(\mathfrak{F})$ for all $\mathfrak{F} \in \Gamma$ implies $y \in B$ for all $B \in B$. This completes the proof.

The author wishes to thank Mr. Gerald Fisher, of the University of Connecticut, for helpful conversations on this subject.

\section{REFERENCES}

1. J. W. Alexander, Ordered sets, complexes, and the problem of bicompactification, Proc. Nat. Acad. Sci. U.S.A. 22 (1939), 296-298.

2. W. H. Gottschalk, Choice functions and Tychonoff's theorem, Proc. Amer. Math. Soc. 2 (1951), 172.

3. J. L. Kelley, General topology, Van Nostrand, Princeton, N. J., 1955.

4. - The Tychonoff product theorem implies the axiom of choice, Fund. Math. 37 (1950), 75-76.

5. R. Rado, Axiomatic treatment of rank in infinite sets, Canad. J. Math. 1 (1949), 337-343.

6. H. Wallman, Lattices and topological spaces, Ann. of Math. 39 (1938), 112-126.

7. L. E. Ward, Jr., $A$ weak Tychonoff theorem and the axiom of choice, Proc. Amer. Math. Soc. 13 (1962), 757-758.

UnIversity of Connecticut 